\title{
Norois
}

Environnement, aménagement, société

\section{De l'identité territoriale au développement local par la restauration et les productions localisées (l'exemple du Cocido de Lalín en Galice)}

From territorial identity to local development by local restoration and localized products (the example of Cocido of Lalín in Galicia)

\section{Román Rodriguez González et José Antonio Aldrey Vázquez}

\section{(2) OpenEdition}

\section{Journals}

\section{Édition électronique}

URL : https://journals.openedition.org/norois/4292

DOI : $10.4000 /$ norois.4292

ISBN : 978-2-7535-2157-5

ISSN : $1760-8546$

\section{Éditeur}

Presses universitaires de Rennes

Édition imprimée

Date de publication : 30 septembre 2012

Pagination : 77-90

ISBN : 978-2-7535-2155-1

ISSN : 0029-182X

\section{Référence électronique}

Román Rodriguez González et José Antonio Aldrey Vázquez, « De l'identité territoriale au

développement local par la restauration et les productions localisées (l'exemple du Cocido de Lalín en Galice) », Norois [En ligne], 224 | 2012, mis en ligne le 30 septembre 2014, consulté le 14 janvier 2022. URL : http://journals.openedition.org/norois/4292 ; DOI : https://doi.org/10.4000/norois.4292 


\title{
De l'identité territoriale au développement local par la restauration et les productions localisées (l'exemple du Cocido de Lalín en Galice)
}

\author{
From Territorial Identity to Local Development by Local Restoration and Localized Products \\ (the Example of Cocido of Lalin in Galicia)
}

\author{
Román Rodriguez González, José Antonio Aldrey Vázquez
}

Département de Géographie, Université de Santiago de Compostela, Plaza da Universidade, 115782 Santiago de

Compostela A Coruña - Espagne (roman.rodriguez@usc.es) (joseantonio.aldrey@usc.es)

Résumé : Cet article décrit le processus de développement territorial vécu par Lalín, localité de Galice intérieure, dans le cadre de la valorisation d'un plat traditionnel des zones rurales : le cocido. Il vise à démontrer la progressive assimilation entre le plat et la localité à travers un processus politiquement planifié de construction d'un discours et d'un imaginaire, depuis la création en 1969 de la Feria del Cocido, et de sa reconnaissance intérieure et extérieure à différentes échelles territoriales. Le texte évalue les stratégies menées et leurs conséquences sur la structuration urbaine, les dynamiques touristiques et de loisirs et l'économique locale. Une attention particulière est donnée aux restaurants de la ville et la relation entre l'offre de ce plat et sa viabilité. En définitive, l'article vise à présenter et à évaluer le rôle des cultures culinaires et de la restauration dans le développement local à Lalín.

\begin{abstract}
This article deals with the process of creating a shared territorial identity between Lalin, a small inland town in Galicia (NW Spain), and one of the traditional culinary products of european rural areas, the hearty pork stew known as 'cocido'. This creation of identity is based in a conscious and human process of construction that began in 1969 with the promotion, still ongoing, of the annual festival baptised with the name Feria del Cocido, which has helped to produce a brand image shared by the territory and the culinary product in question. An assessment is made of the promotional strategies adopted and their effects on the structure of the local economy, with particular attention being paid to the network of restaurants in Lalin and the interrelation between having this dish on the menu and their viability. In short, the intention is to evaluate and highlight the role of gastronomy and the restaurant industry as an element of local development in Lalin.
\end{abstract}

Mots clés : culture culinaire - image de marque - marketing territorial - restaurants - développement local - tourisme - Lalín (Galice)

Keywords: culinary culture - brand image - territorial marketing - restaurants - local development - tourism - Lalin (Galicia) 


\section{INTRODUCTION}

La gastronomie et les systèmes et pratiques culinaires qui étaient de simples particularismes régionaux ou locaux hier ont atteint aujourd'hui une grande reconnaissance en s'identifiant comme patrimoine culturel immatériel doté d'une fonction identitaire (Csergo et Lemasson, 2009; Urry, 2002). C'est l'exemple de la France qui en 2010, a vu sa gastronomie et plus précisément le « repas gastronomique » reconnu au titre de Patrimoine Culturel Immatériel de l'Unesco. Cette distinction est due au fait que la cuisine est une pratique sociale coutumière qui bénéficie d'une nette « représentativité » aux yeux des Français. Elle fait partie de leur identité et de leur goût culinaire. Toutes proportions gardées, le cas de Lalín et de son plat phare, le cocido, est également à la base de la construction d'une identité culinaire et d'une saveur locale. À partir d'un plat traditionnel des familles paysannes, le pouvoir local a poursuivi une politique de développement touristique gastronomique de manière consciente et programmée, associée à l'activité d'élevage et à l'industrie agroalimentaire.

Il convient donc de réfléchir sur ces aspects, sur la façon dont se sont développées ces cultures culinaires, sur quelles connaissances, habitudes et usages elles ont été socialement établies. On peut d'ailleurs évoquer d'éventuels métissages car ces pratiques alimentaires sont parfois le patrimoine commun à plusieurs peuples (c'est le cas du « cocido galicien »- sujet de notre article - qui a une base commune dans divers peuples européens) bien que certains offrent une originalité et une identité qui leur sont propres (Fumey et Etcheverria, 2009). Cette approche de la cuisine comme phénomène de grande valeur culturelle est intimement liée à son intérêt touristique croissant : elle est ainsi devenue une ressource touristique et culturelle de première valeur (Armesto et Gomez, 2004; Boniface, 2003; Calabuig et Ministral, 1999; Cazes, 1992). Mais audelà du tourisme culinaire ou gastronomique, nous devons évoquer le tourisme gourmand (Etcheverria, 2010, 2009; Lemasson, 2006), celui des gens intéressés non seulement par les aspects culturels liés à l'offre gastronomique mais aussi qui savourent les plaisirs culinaires, autrement dit, qui sont de la bonne table. Ce type de tourisme a été initié par ceux qui possèdent une connaissance avancée de la tradition culinaire. Ils veillent sur une pratique vivante des rites gastronomiques qu'ils transmettent en renforçant les liens sociaux et affectifs avec les lieux visités.

La cuisine est une ressource touristique appréciée non seulement par sa valeur intrinsèque mais aussi par son caractère symbolique puisqu'elle agit comme un identifiant des peuples et des territoires (Armesto et Gomez, 2006). Aussi s'est-elle s'est affirmée ces dernières années comme un puissant élément générateur d'identités territoriales. En ce sens, on doit considérer que l'identité acquiert une importance notoire dans une société globalisée où la culture a subi en parrallèle un processus de standardisation (López et Lois, 2007; Terkenli, 2002). Cette évolution vers l'homogénéisation est à rattacher au processus de « mac donalisation » et « coca-colatisation » ou à l'impérialisme culinaire nord-américain (Ritzer, 1998) et a entraîné en réaction un désir d'«authentique » et de « traditionnel » (Moulin, 2000; Maby, 2002). Pour certains territoires, cette réponse a généré une véritable dynamique de développement local basé, entre autre, sur le restaurant et le service de restauration.

Cette ressource s'est également manifestée par un attrait de touristes bien informés de l'existence d'un restaurant distingué dans des guides prestigieux, connaisseurs d'un réseau de gites servant des repas traditionnels très souvent reliés à des offres en tourisme rural et par la présence de fêtes gastronomiques (comme c'est le cas dans notre article) devenues l'élément moteur du développement local (Csergo et Lemasson, 2009).

Quelle importance donne-t-on, dans une petite localité de l'arrière pays galicien nommée Lalín, au goût et à la restauration associée à la valorisation d'un plat «traditionnel » des campagnes galiciennes : le cocido $^{1}$ ? Cette recette traditionnelle dont les ingrédients ont peu évolués lors des deux derniers siècles a récemment fait l'objet d'un effort particulier pour la recherche d'une meilleure qualité des produits qui la composent, notamment pour l'optimisation de la chaîne de production de la viande porc, base du cocido, depuis l'élevage jusqu'à la table. Au niveau de la région, le pays de Deza, (figure 1), connaît

1. Le « cocido galicien » : variété de porc fumé, de viandes bouillies et cuites avec des pommes de terre, des légumes et du chorizo. Il s'agit d'une recette traditionnelle qui lors de ces deux derniers siècles n'a subi guère de modifications dans ses ingrédients. 
une forte hausse de la production de viande salée et de la charcuterie. On assiste en parallèle à une identification croissante de ce mets de la part des producteurs et de surcroît de la plupart des habitants de la région. Le cocido est devenu un élément patrimonial, une partie de la culture locale ayant des retombées sur le développement local.

Au cours de cet article, nous étudierons les répercussions de ce mets sur le développement local d'une région de Galice, le Deza et son centre Lalín. Nous ferons une analyse qualitative du processus de construction de l'identité culinaire du village et de la région à partir de ce plat qui contient essentiellement de la viande de porc. Nous verrons l'essor d'activités économiques telles la restauration, l'élevage, l'industrie agroalimentaire et le tourisme. Cette approche qualitative débouchera sur l'analyse des effets directs sur l'économie de la région. Pour conclure, nous présenterons une série de résultats du processus analysé.

\section{LA CONSTITUTION ORGANISÉE ET CONSCIENTE DU DISCOURS ET DE L'IMAGINAIRE «LALÍN- COCIDO " PAR OU POUR LES RESTAURANTS}

L'identification d'une cuisine de qualité est liée habituellement à l'existence d'aliments emblématiques, présentant fréquemment des signes de distinction et de protection liés à l'origine géographique et à la qualité : appellation d'origine contrôlée, indication géographique protégée, appellations spécifiques, etc. (Armesto et Pazos, 2010).

Dans la société actuelle, les valeurs associées aux singularités de chaque territoire acquièrent une valeur différentielle et constituent une opportunité favorable de développement local (Albet, 2001). La cuisine et la restauration montrent un rapport étroit avec les caractéristiques de l'espace géographique dans lequel elles se développent, tout particulièrement pour les offres à caractère populaire. Les caractéristiques agronomiques de chaque espace donnent une primauté à certaines matières primaires agraires, d'élevage ou liées aux activités de la pêche. Unies à la séquence historique d'utilisation, de transformation et de consommation de ces aliments, il existe la création d'un lien direct entre les produits alimentaires et le territoire. En ce sens, les restaurateurs de Lalín ont développé l'utilisation de produits locaux pour l'élaboration du cocido. Cette circonstance a permis de renforcer l'identification entre le territoire de Lalín et ce plat, offre traditionnelle qui a évolué jusqu’à la situation actuelle. Ainsi, l'identification de territoires singuliers et de leurs produits spécifiques fait émerger une vraie image de marque. Pour certains espaces, l'identité et le rayonnement sont nettement liés à une cuisine ou une spécialité culinaire déterminées (Moulin, 2000).

Cependant, la rencontre entre la petite localité galicienne de Lalín (figure 1) et un plat singulier enraciné dans la cuisine populaire, le cocido, ne répond pas uniquement à des caractéristiques géographiques singulières et spécifiques mais aussi au développement d'une stratégie de promotion et de marketing territorial. Elle naît de façon volontaire et va conduire à la mise en place d'actions parfaitement planifiées dont l'évolution va favoriser l'émergence d'un secteur économique important lié à la restauration et à la transformation et commercialisation de produits locaux du secteur primaire.

Ce processus d'identification a tout d'abord été activé par les autorités politiques locales, premiers promoteurs de la Feria del Cocido. Plus tard cette célébration sera diffusée dans l'ensemble de la société et c'est ainsi que plusieurs associations (sportives, gastronomiques...) renforceront l'identification Lalín-Cocido à partir de l'organisation de manifestations de promotion. Les propriétaires locaux de restaurants y ont d'ailleurs joué un rôle important en organisant des journées gastronomiques, en présentant leurs produits et en enrichissant la qualité de leur offre. Parallèlement, les fabricants de produits pour l'élaboration du cocido (charcuterie et viande de porc) ont évolué et leur production s'est élargie. Il est évident que le cocido est devenu un élément de la dynamique de développement local pour Lalín et sa région, notamment à partir des années 1990 où la célébration devient importante et profite d'une grande diffusion grâce à l'action directe des dirigeants locaux ainsi que des restaurateurs.

Si dans un premier temps il n'y avait aucun rapport entre cette fête gastronomique et les traditions du Carnaval, un rapprochement très étroit s'est fait peu à peu. C'est sans doute parce que le cocido est un plat typique en Galice qui plus est, consommé pendant cette période de l'année. C'est ainsi que 


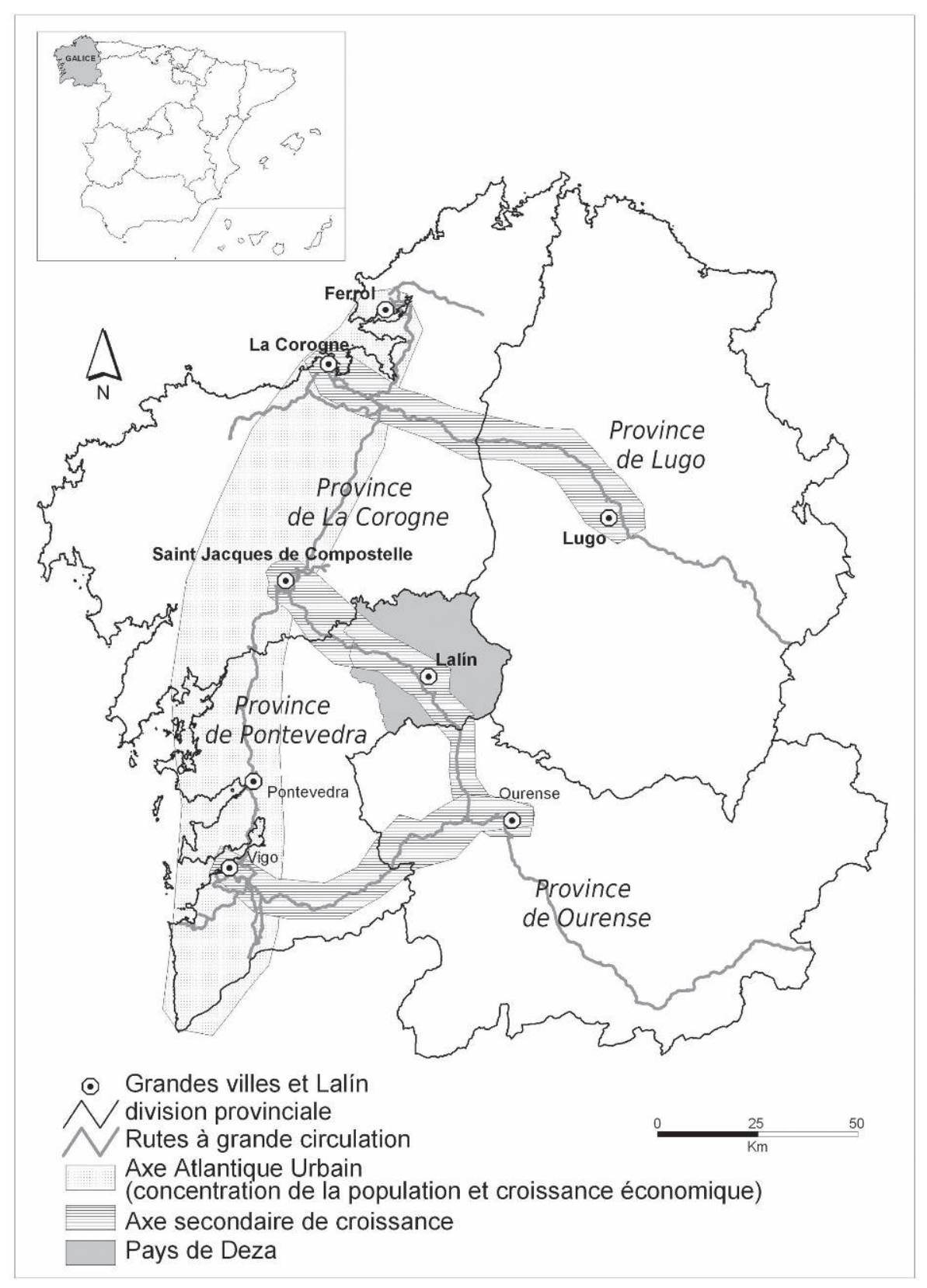

Figure 1 : Situation géographique de Lalín

Lalín

Geographical location of l'on a fait coïncider les dates de la Foire $d u$ Cocido et la fête du Carnaval : Lalín est alors devenu un repère territorial marqué pendant cette période.

Parallèlement, le processus d'urbanisation et de développement qu'a connu Lalín depuis les années 1960 est à mettre en relation avec la consolidation de l'image de marque du cocido et par conséquent à l'accroissement de l'activité de restauration. Cette petite ville dynamique située au centre de la Galice a renforcé ses actions économiques et urbaines grâce à sa situation favorable au carrefour de voies de communication, point de rencontre et de transition entre l'espace urbain littoral au dynamisme marqué et l'intérieur à forte empreinte agricole (Aldrey et Lois, 2010). Cette situation géographique est un facteur clé pour comprendre sa fonction d'axe central par rapport à la région de Deza, espace intégré, et toutes les communes de Lalín, d’Agolada, Dozón, Rodeiro, Silleda et Villa de Cruces (figure 1) où résident environ 45000 habitants. Il s'agit originellement d'un espace agricole, orienté vers l'élevage, qui a modifié sa structure de production par la création 
d'emplois industriels et tertiaires (essentiellement à Lalín). Le noyau urbain actuel de cette petite ville compte plus de 12000 habitants. Il a connu une très forte croissance en lien avec le processus général d'urbanisation et de modernisation de la Galice. Dans ce contexte, Lalín montre une croissance urbaine remarquable puisqu'elle ne comptait que 1000 habitants en 1960.

Ce développement socio-économique est fondé sur la valorisation des ressources endogènes et sur la consolidation d'une base agro-industrielle diversifiée qui reposent sur une économie agricole, orientée vers l'élevage bovin laitier et porcin. Le modèle de développement repose sur l'existence d'un nombre important d'entreprises locales (spécialisées dans les secteurs de la confection, l'agro-industrie de transformation) et sur une base tertiaire de plus en plus élargie et spécialisée (Alonso, 2000; Rodríguez González, 2000). Lors de ces dernières années, cette situation a permis l'apparition d'un marché du travail à caractère urbain, essentiel pour comprendre les actions actuelles de développement de cette petite localité. La population du noyau urbain augmente très rapidement entre chaque période intercensitaire, cette hausse étant basée sur l'arrivée de nouveaux habitants venant d'autres localités proche (de la région de Deza), des émigrants galiciens revenus au pays et, plus récemment, des immigrés issus essentiellement d'Amérique Latine. Le progrès fonctionnel est aussi très net et lisible dans l'augmentation du nombre de licences commerciales : 107 en 1960 et 671 en 2009. Soulignons aussi l'apparition d'activités industrielles dans le marché de l'emploi actuel : $20 \%$ de la population active se consacre aux activités agricoles alors qu'en 1960, $90 \%$ de cette population travaillait dans l'agriculture.

Dans ses travaux de recherche, R. Rodríguez González (1994) a montré que les actions de développement précédentes, à caractère rural marqué, subissent un changement à la fin des années 1960. Ce moment coïncide avec la première édition de la Feria del Cocido (en 1969) et le démarrage du secteur de la restauration associé à ce mets de la diète rurale galicienne. Parallèlement, la restauration a subi une transformation à la fois quantitative et qualitative. En 1960, il n'y avait aucun restaurant alors qu'aujourd'hui on en compte une trentaine. On a ainsi assisté à une adaptation aux changements sociaux et économiques et on est passé de petites auberges très modestes à un réseau d'établissements spécialisés dans une offre culinaire basée sur le cocido. Cela a supposé pour Lalín la création de nombreuses offres d'emploi de salariés et d'une augmentation progressive de la base des salaires. Tout cela a promu la modernisation fonctionnelle de la petite ville.

La force de l'identification «Lalin-Cocido » et l'effet de dynamisation sur l'économie locale sont à relier au fait qu'elle a été créée et soutenue par les pratiques de la société locale et organisée par les autorités municipales. Ce plat issu du monde agricole galicien est ainsi devenu une spécialité urbaine spécifique. Lors de ces dernières années, le travail des restaurateurs locaux a favorisé la qualité de leur production finale et l'image de marque de Lalín et de son offre gastronomique principale.

Le cocido gallego entre dans le groupe des mets de la diète européenne rurale. Dans la plupart de ces zones géographiques, ce plat est en effet associé à une diète de subsistance. Il se compose de légumes de saison, de pommes de terre, de pois chiches ou de haricots blancs (selon les régions), de viande de porc, de poulet ou de vache conservée à l'ancienne, fumée ou salée. Son nom varie aussi : dans la chaîne Cantabrique, il s'appelle «pote », en Aquitaine « pot$a u-f e u$ » et dans le Portugal «cozido». Toutefois, son élaboration, son mode de cuisson et ses ingrédients sont similaires pouvant varier selon les matières premières de chaque zone ou selon les coutumes (Gonzalez Alén, 2008).

L'identification «Lalín-Cocido » se construit dès 1969 avec la première édition de la Feria del Cocido (fig. 2). Le maire Luis González Taboada est le premier promoteur de l'organisation de l'événement qui a contribué à diffuser et à promouvoir Lalín comme une capitale gastronomique (Etchevarria, 2009). Il eut lieu en plein hiver, le dimanche avant le carnaval. Parallèlement, et en relation avec l'origine agricole du plat, un grand marché à la viande de porc et aux produits laitiers ainsi qu'une foire au bétail et aux machines agricoles se tinrent. Ces marchés et foires apportèrent des retombées économique considérables à la société de Lalín. En outre, lors de cette première édition, fut lancée la figure du crieur public «pregonero » : personnage célèbre venant faire un discours gastronomique louant la qualité et la richesse culturelle et sociale que constitue le cocido. Ajoutons à cela, l'importance visuelle de l'af- 
fiche qui symbolise d'une façon artistique l'intérêt pour la diffusion du mets (fig. 2 et 3 ).

D’après le chroniqueur local, González Alén (2008), le succès de cette première édition et les bénéfices financiers pour Lalín ont incité au maintien de cet événement, selon le même mode d'organisation. Depuis les premières éditions, certes plus humbles et à rayonnement local, le nombre de visiteurs n'a cessé de croître. La municipalité de Lalín estime aujourd'hui la fréquentation à 50000 personnes. La promotion basée sur le cocido a entraîné la création d'une image de marque « authentique » et un lien étroit, par le truchement de ce mets élaboré dans toute la Galice intérieure, entre cette ville et la région galicienne.

À partir de là, la qualité et la réputation qualitative des viandes de porc et des charcuteries de Lalín,

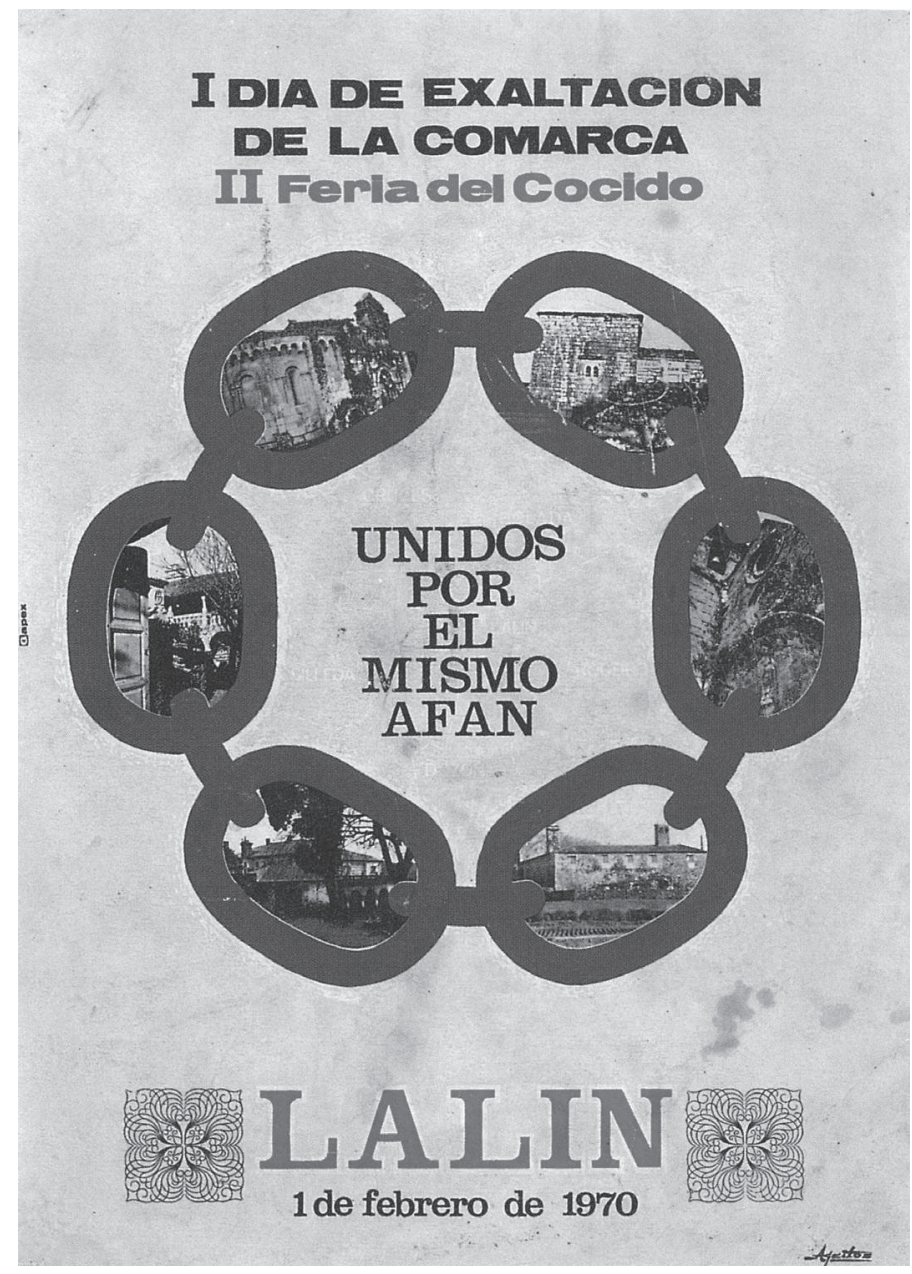

Figure 2 : Affiche de la $2^{\mathrm{e}}$ édition de la Feria del Cocido (1970) Poster announcing the $2^{\text {nd }}$ edition of the Feria do Cocido (1970) préparées selon un traitement artisanal, ont contribué à la diffusion de la reconnaissance du cocido sur la zone côtière de Galice la plus peuplée et dont le revenu par habitant est le plus élevé. En venant à Lalín, les touristes gourmands de ces premières éditions ont favorisé le développement du secteur de la restauration et ont accru la vente des produits issus pour la plupart des excédants de production des familles campagnardes. Depuis le début, on assiste à une consolidation des retombées financières pour le secteur de la restauration locale et pour la population rurale qui a su structurer la vente de produits locaux pour un nombre croissant de visiteurs. La reconnaissance qualitative des produits et la naissance d'un marché ont facilité aussi l'apparition d'un nouveau secteur de production agroalimentaire spécialisé dans l'élaboration de charcuteries et de la vente de produits dérivés du porc.
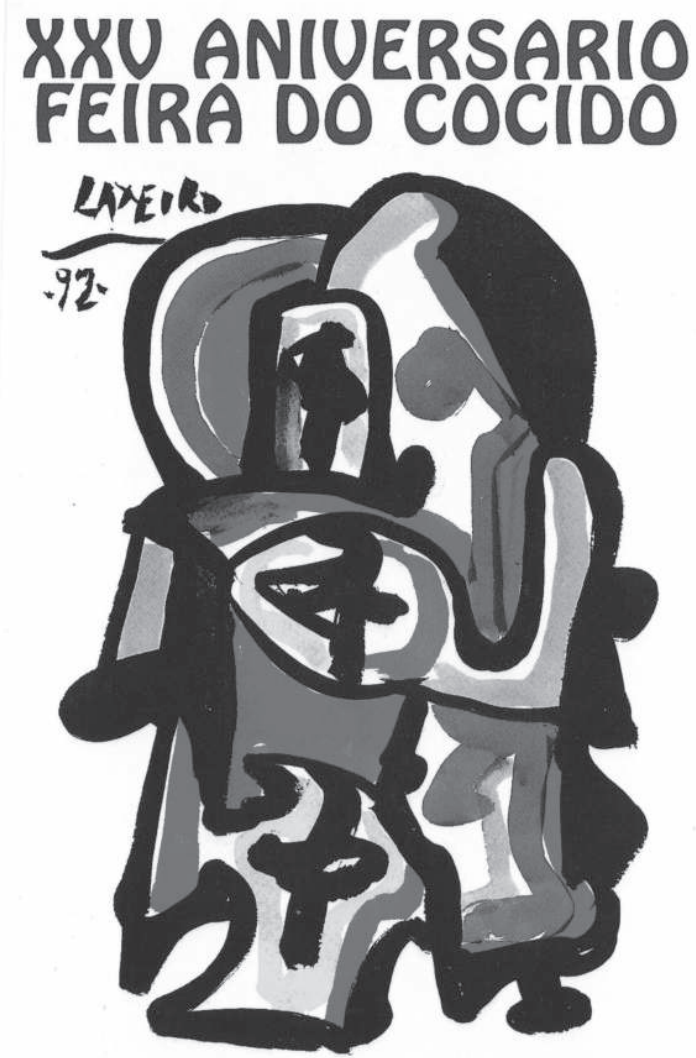

\section{LALIN - 14 DE FEBREIRO - 93}

Figure 3 : Affiche de la $25^{\mathrm{e}}$ édition de la Feria del Cocido (1993). Euvre de Laxeiro, un des peintres les plus importants de Galice du $\mathrm{XX}^{\mathrm{e}}$ siècle

Poster announcing the $25^{\text {th }}$ edition of the Feira do Cocido (1993), the work of Laxeiro, one of Galicia's most outstanding $20^{\text {th }}$ century artists 
Depuis 1969, la succession des équipes municipales n'a entrainé aucun changement dans l'organisation de l'événement. En 1979, lors des premières élections démocratiques en Espagne, le nouveau maire José Cuiña Crespo prend le relais pour cette célébration qui fête son douzième anniversaire. À partir de 1990, le maire José Crespo Iglesias donne l'élan décisif à la Feria del Cocido, élément de dynamisation de l'économie locale essentiellement dans les secteurs de la restauration et de la commercialisation de produits porcins. L'action de promotion menée à bien par les autorités municipales est le facteur essentiel de renforcement et d'accroissement d'une offre gastronomique comme le cocido On a gardé l'habitude de la réalisation l'affiche, permettant ainsi à des peintres de renom régional et national de confectionner des œuvres d'art collectionnées dans l'importante pinacothèque municipale. On a aussi institutionnalisé le crieur public « pregonero » en invitant des personnages du monde de la culture, de la politique, du sport, du journalisme, des acteurs (fig. 4). Ils ont aidé, par leur popularité, à donner un effet médiatique et de diffusion remarquable sur différents medias et supports de communication.

Depuis sa création, outre l'élan municipal, la promotion de la Feria del Cocido est basée sur la qualité de l'offre gastronomique des restaurants qui se sont efforcés d'améliorer la qualité du plat. Lors de ces dernières décennies, on assiste aussi à une action de divulgation dans les médias. L'intérêt mutuel d'associer la stratégie de promotion territoriale promue par la municipalité à l'offre du cocido et celui des restaurants qui augmentent leur chiffre d'affaires a contribué à consolider l'identification «Lalin-Cocido».

Ce faisant, on a également cherché à donner un nouvel élan à la Feria del Cocido. Durant le weekend précédant le carnaval, dans les années 1980, de nouvelles activités touristiques furent organisées : un défilé de carrosses, des fanfares, des orchestres qui donnèrent à l'événement un caractère beaucoup plus festif. En 1994, le « Prix de Gastronomie » et le «Gala Gastronomique de Galice » sont institués. Les "Prix de Gastronomie », en relation avec les principales associations gastronomiques de Galice, décernent des récompenses, dans différentes catégories, à des professionnels du journalisme gastronomique et culturel, à des restaurants de renom et à des institutions qui ont un rapport étroit avec la promotion de la cuisine et du cocido. La remise de ces prix a lieu lors d'une émission télévisée diffusée dans toute la Galice avec la participation de personnages du monde de la musique, des humoristes nationaux et internationaux. La capacité de rayonnement atteint une nouvelle échelle et l'identification «Lalín-Cocido » est reconnue d'un très large public. En 2000, nait la «Encomienda del Cocido» (Commanderie du cocido), institution qui regroupe des acteurs ayant eu un grand rôle dans la promotion de Lalín et du cocido dans différents domaines (culturel, politique, monde de l'entreprise, etc.). La «Encomienda» est une association sans but lucratif qui est formée par des personnalités espagnoles et étrangères de divers secteurs (politique, culture, cuisine, université, etc.) chargés de diffuser la fête gastronomique à l'étranger. Elle compte aussi sur des personnages locaux reconnus dans leurs domaines professionnels et qui ont créé un sentiment d'identification pour une incitation et une reconnaissance. La date choisie pour cet événement, le dimanche avant le Mardi-Gras, a engendré une sensation de festivité hivernale, prélude des festivités du carnaval. Ce motif fut au cours du temps un élément de plus pour renforcer la popularité de cette célébration.

Ainsi, des politiques espagnols de tous bords, des artistes consacrés (Joaquin Sabina, Julio Iglesias), des restaurateurs au prestige reconnu dans le guide Michelin comme Toñi Vicente ou Karlos Arguiñano (figure 5) font partie de cette Encomienda del cocido

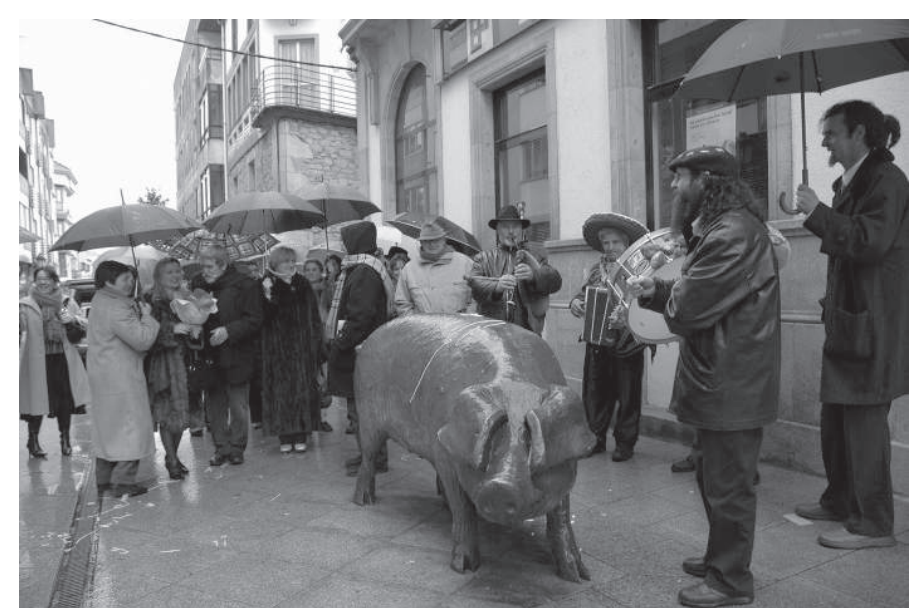

Figure 4 : Célébration du Cocido das Artes avec des représentants de la culture galicienne of Galician culture 


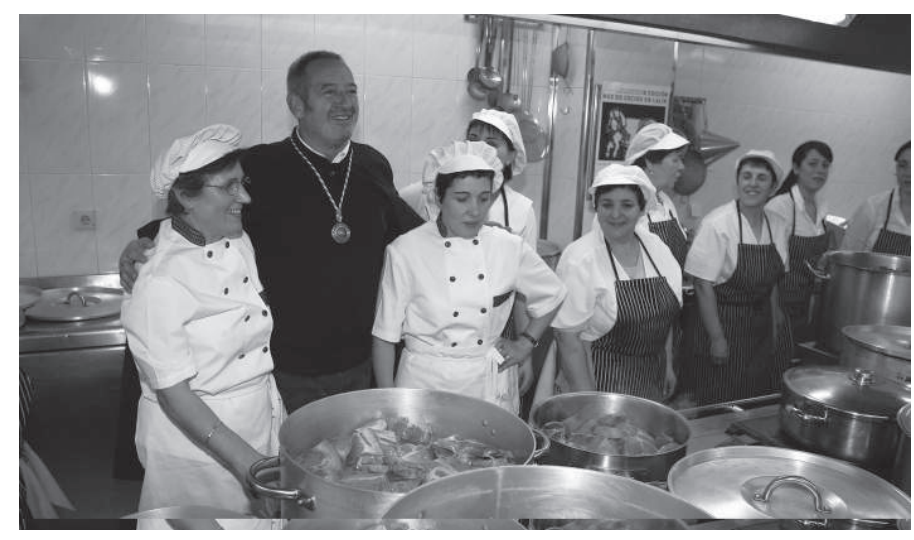

Figure 5 : Karlos Arguiñano, un des plus célèbres cuisiniers d'Espagne. Ici dans la Feria del Cocido

Cocido

Karlos Arguiñano, one of Spain's best-known chefs, at the Feria del

qui est devenu un outil puissant de rayonnement pour le rendez-vous gastronomique de Lalín.

Parallèlement, on organise à partir de cette date des cocidos à l'international, dans des capitales où les émigrants galiciens sont nombreux (Buenos Aires, Caracas, Hambourg, Andorre...) et dans de nombreuses villes espagnoles qui ont institué le cocido comme une pratique sociale en hommage à Lalín et à la Galice. (Madrid, Las Palmas, Barcelone...). Des échanges avec d'autres localités disposant de spécialités culinaires de cocido (Astorga en León, Potes en Cantabrie...) se développent tout comme des journées de promotion, dans les restaurants de Lalín, où sont mis en valeur des spécificités culinaires de cocidos d'autres régions. Ajoutons à cela la récupération de gestuelles ethnographiques tel l'abattage de porcs, l'organisation d'événements sportifs et une présence médiatique constante.

Nous pouvons donc conclure que l'identification "Lalin-Cocido» est un produit social construit. C'est le résultat d'un processus organisé et conscient de création d'une image de marque à partir de la célébration d'une série d'actes et d'activités de promotion qui ont entraîné une identification directe. Cette identité est un support essentiel au réseau de restaurants locaux et au secteur de la transformation et de la commercialisation des viandes. Il faut souligner l'effet social important : la création d'un grand nombre de postes de travail dans toute la région de Lalín. Ce qui est remarquable, c'est la capacité des acteurs de la ville de Lalín à s'approprier une préparation culinaire originaire de toute la
Galice, à constituer autour d'elle un discours et un imaginaire identificatoires afin d'en faire la promotion comme une ressource fondamentale du développement local.

Pour autant, les conséquences économiques, sociales et culturelles directes et indirectes du cocido de Lalín restent difficiles à évaluer. S'il est facile de déceler les effets sur la dynamique du réseau de restaurants locaux et sur la création d'emplois associés à la restauration, il est moins aisé d'obtenir une information quantitative fiable sur la commercialisation et la transformation de viandes et d'autres matières premières, ni sur les effets liés à la consommation des touristes dans les commerces et services locaux. L'observation empirique est complexe et peu fiable, cependant la hausse des ventes, y compris dans le commerce textile de Lalín, est confirmée $e^{2}$.

Ce processus d'accroissement de l'activité de restauration à partir du cocido ne peut être dissocié des changements sociaux et territoriaux qui ont eu lieu en Galice dans les dernières décennies. Différents spécialistes en sciences sociales (anthropologistes, économistes, sociologues, etc.) ont analysé le processus d'urbanisation pour le territoire galicien à partir des années 1960, basé sur un essor rapide d'un monde rural traditionnel, initialement renfermé sur lui-même, sans à peine d'échanges ni d'informations et reposant sur une économie agraire de subsistance. Les symboles de cette identité culturelle ont disparu progressivement tandis que la société rurale s'est ouverte et, hormis certains cas, elle a été capable de s'adapter aux modèles culturels urbains. C'est le cas de la Feria del Cocido et de la promotion de l'activité gastronomique associée, devenue, lors de ces dernières années, une référence touristique, en particulier pour les habitants des villes galiciennes. Certaines activités ont eu un rôle essentiel dans la construction sociale de ce produit touristique, qu'elles soient culturelles, ethnographiques, journalistiques ou de loisirs.

En ce sens, un mets à caractère familial, associé à une économie rurale de subsistance, est aujourd'hui, grâce à une grande activité de promotion, un produit de restauration sur lequel repose un modèle

2. On doit tenir compte de l'activité des entreprises de Lalín dans le domaine de la confection qui possèdent des marques reconnues dans la région et en Espagne, ce qui renforce l'attrait de l'offre et entraîne un complément dans les dynamiques de consommation. 
de développement local et touristique. Il attire une clientèle essentiellement urbaine qui associe le cocido à un retour aux origines rurales de la société galicienne, alors que la population rurale à Lalín et sa région ne fait pas partie de la clientèle habituelle des restaurants puisque ce plat est préparé fréquemment dans ces familles avec les produits issus de l'unité familiale. En ce sens, l'urbanisation progressive de la société galicienne des dernières décennies a été un facteur qui a favorisé le potentiel d'intérêt de cette célébration gastronomique.

\section{Pérenniser et désaisonnaliser LES EFFETS SUR LE DÉVELOPPEMENT LOCAL : LE MES DEL COCIDO}

Les efforts pour augmenter et étaler les retombées économiques et sociales de la Feria del Cocido se matérialisent dans la promotion du Mes del Cocido (le mois du cocido), du 15 janvier au 14 février. Il s'agit d'une nouvelle initiative de promotion à laquelle participent de façon coordonnée plus de 30 restaurants qui proposent le cocido dans leur menu, en particulier pour les groupes et sur commande. Cette initiative mise en place par les autorités municipales est généralisée à toute la Galice. Il attire de nombreux touristes qui, d'après les estimations des hôteliers-restaurateurs, atteignent plus de 100000 personnes, (d'après les estimations du nombre des visiteurs réalisées par la Municipalité de Lalín). L'offre de capacité dans les restaurants s'élève à 3700 places qui sont couvertes sur réservation durant les week-ends du Mes del Cocido. L'un des objectifs affichés est de renforcer la capacité d'attraction touristique et de désaisonnaliser l'activité touristique. Dans tout ce processus, le rôle des restaurants locaux a été primordial. Ils se sont impliqués pleinement dans la stratégie générale d'élan de la Feria del Cocido. Ils ont apporté leur savoir-faire et ont amélioré l'offre qui est la base de la promotion de cette célébration gastronomique. D’après les estimations des acteurs du secteur de la restauration de la municipalité de Lalín, cette initiative permet la vente de 130000 à 150000 cocidos par saison. Si nous tenons compte du prix unitaire de ce plat qui est d'environ 30 euros, (comme en attestent les documents de promotion), les recettes se situent entre 3900000 et 4500000 euros. En outre, les effets positifs se ressentent aussi dans la structure commerciale et dans l'activité de commercialisation de matières premières (figure 6). L'ensemble entraîne un élan considérable de l'économie locale à partir des synergies que la valorisation touristique du cocido autorise entre les restaurants, les usines de charcuterie et le secteur commercial en général.

La quantification détaillée et exacte des effets directs est bien évidemment très complexe par le manque de données statistiques objectivables et l'absence de sources d'informations officielles. Toutefois, vu les données obtenues de l'information fournie par les propriétaires des restaurants, l'impact sur l'économie locale est indiscutable.

En 2010, Lalín compte un grand nombre d'entreprises familiales artisanales liées à la transformation et à la commercialisation de produits dérivés de la viande porcine. D'après des sources municipales officielles, on y compte 31 boucheries et 8 usines de charcuterie qui occupent une place importante dans le marché grâce au renforcement de «l'image de marque ». L'entreprise pionnière à grande envergure Embutidos Lalinenses [www.embutidoslalinense.com] offre une forme de mécénat pour la promotion de la Feria del Cocido et présente un chiffre d'affaires de plus de 7 millions d'euros et un effectif de plus de 20 travailleurs directs. Le cocido apporte aussi aux exploitants agricoles une source complémentaire de revenus puisque de nombreux restaurants achètent directement les animaux (sacrifiés et transformés dans des abattoirs autorisés) aux éleveurs qui pro-

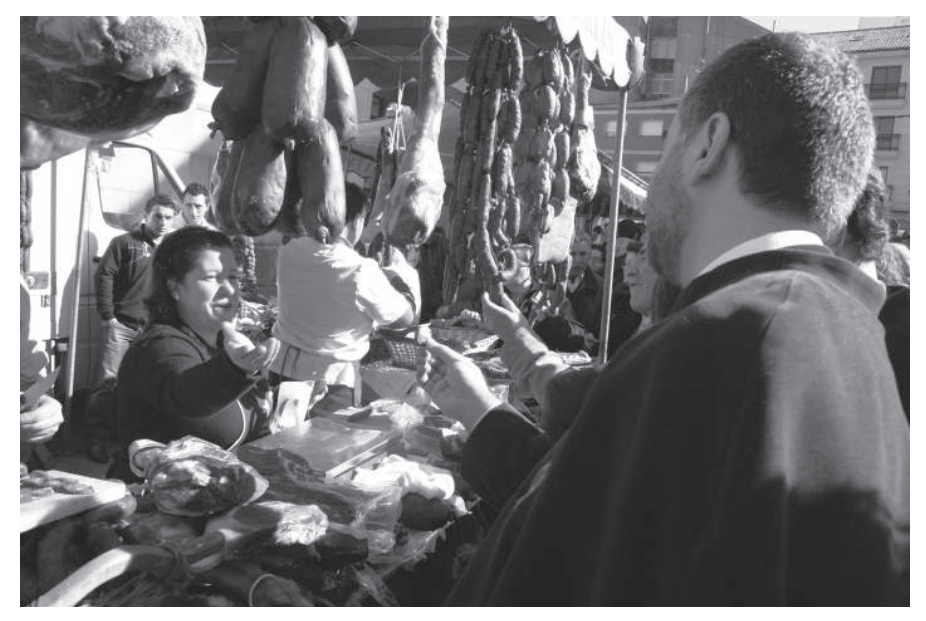

Figure 6 : La vente de produits de charcuterie de qualité participe au développement local à Lalín

Lalin

The sale of top-quality cured meats favours local development in 
posent un produit de grande qualité par le choix de l'alimentation animale et le soin accordé aux animaux, très différent de ce qui existe dans les exploitations industrielles. Les informations fournies par des restaurateurs locaux permettent d'estimer une recette supplémentaire de 4000 à 6000 euros qui vient compléter les finances domestiques.

De plus, l'image de marque est renforcée per une meilleure perception chez les consommateurs de la qualité des produits de viande et de charcuterie de Lalín. La réussite économique de la promotion du cocido comme emblème gastronomique est ainsi renforcée. Elle rapporte de larges bénéfices aux restaurants, au commerce local et aux fabriques de charcuterie et boucherie qui touchent un grand nombre de familles rurales des alentours de la petite localité. C'est cette image acquise par les restaurants de Lalín et la qualité de leurs viandes qui est le facteur essentiel de différenciation de l'offre des entreprises locales. Elles ont développé l'identification de leurs produits à partir et dans le territoire local. Cette union a favorisé l'intérêt pour les usines de viandes élaborées et la vente sur les marchés urbains. L'image de qualité territoriale est ainsi divulguée progressivement et on assiste à une plus grande compétitivité des fabricants locaux.

Selon l'enquête réalisée en 2010, la capacité de création d'emplois, en tenant compte de la saisonnalité caractéristique du secteur de la restauration, se situe autour de 350 à 400 emplois directs. On peut y ajouter 125 à 150 postes liés à la transformation et à la commercialisation de produits de viande associés au cocido. L'importance de cette initiative dans la vie économique locale est donc considérable dans une ville dont la population active compte environ 7000 travailleurs.

Parmi les 31 restaurants (des 58 qui existent à Lalín) qui participent au Mes del Cocido en 2011 nombreux sont ceux qui sont gérés par des restaurateurs d'origine locale. Seuls 3 établissements, installés dans les années 2000, sont la propriété d'entreprises externes spécialisées dans l'offre de services d'hôtellerie et restauration. Nous sommes donc face à une activité nettement endogène qui est basée non seulement sur la valorisation des produits alimentaires locaux mais aussi sur la consolidation des entreprises pilotées par les acteurs locaux qui ont su participé et tirer profit de l'opportunité de la création d'un imaginaire autour du cocido.
Pour Lalín, la répercussion des effets du phénomène gastronomique autour du cocido touche donc le marché du travail par la création d'emploi dans le secteur de la restauration et par la captation des ressources économiques issues de l'émigration. En effet, comme toutes les zones intérieures galiciennes, Lalín a subi dans les années 1950 et 1960 une migration intense qui s'est également ressentie dans la création des restaurants. Sur les 31 établissements cités, 13 d'entre eux sont la propriété d'anciens émigrants qui ont souhaité à leur retour ouvrir un restaurant. Ils ont été ouverts dans les années 1980. À la base de ce mouvement de création réside le fait que ces anciens émigrants ont pour la plupart travaillé dans la restauration dans le pays d'accueil et, à leur retour, ont cherché à tirer profit de cet apprentissage professionnel. Ce mouvement s'inscrit dans celui plus large de dynamisation et d'amélioration du niveau de vie de la société espagnole qui se traduit par une hausse du niveau de revenus et de capacité de consommation. Ainsi, l'existence d'un marché potentiel accru a provoqué la multiplication des restaurants. Ils constituent des lieux incontournables de la promotion et de la pérennité de la Feria del Cocido. Pour ce qui est des autres restaurants, il s'agit d'anciennes auberges à caractère traditionnel qui ont peu à peu augmenté leurs chiffres d'affaire et transformé leurs physionomie et la qualité de leurs offres culinaires. Certains sont d'ailleurs pionniers dans l'offre du cocido et sont à l'origine de l'identification «Lalin-Cocido».

Le caractère traditionnel des restaurants d'aujourd'hui spécialisés dans l'offre de cocido apparaît aussi dans leur localisation dans l'espace urbain. Sur la carte ci-dessous (figure 7) on peut remarquer que les restaurants se trouvent dans les rues centrales de la ville les plus anciennes. Ce qualificatif ne « traditionnel » ne signifie pas pour autant que ces restaurants ne se sont pas impliqués l'évolution de l'offre gastronomique. Bien au contraire, certains restaurants «traditionnels » ont réalisé des innovations dans l'offre de ce produit gastronomique tout aussi bien dans sa préparation que dans sa présentation. Dans certains cas, on offre même de nouvelles recettes qui ont pu intensifier le rayonnement de «Lalín-Cocido».

Loin d'être exhaustive, l'enquête réalisée par nos soins auprès de 3 restaurants (dont on ne divulguera pas le nom pour des raisons de confidentialité) sur 


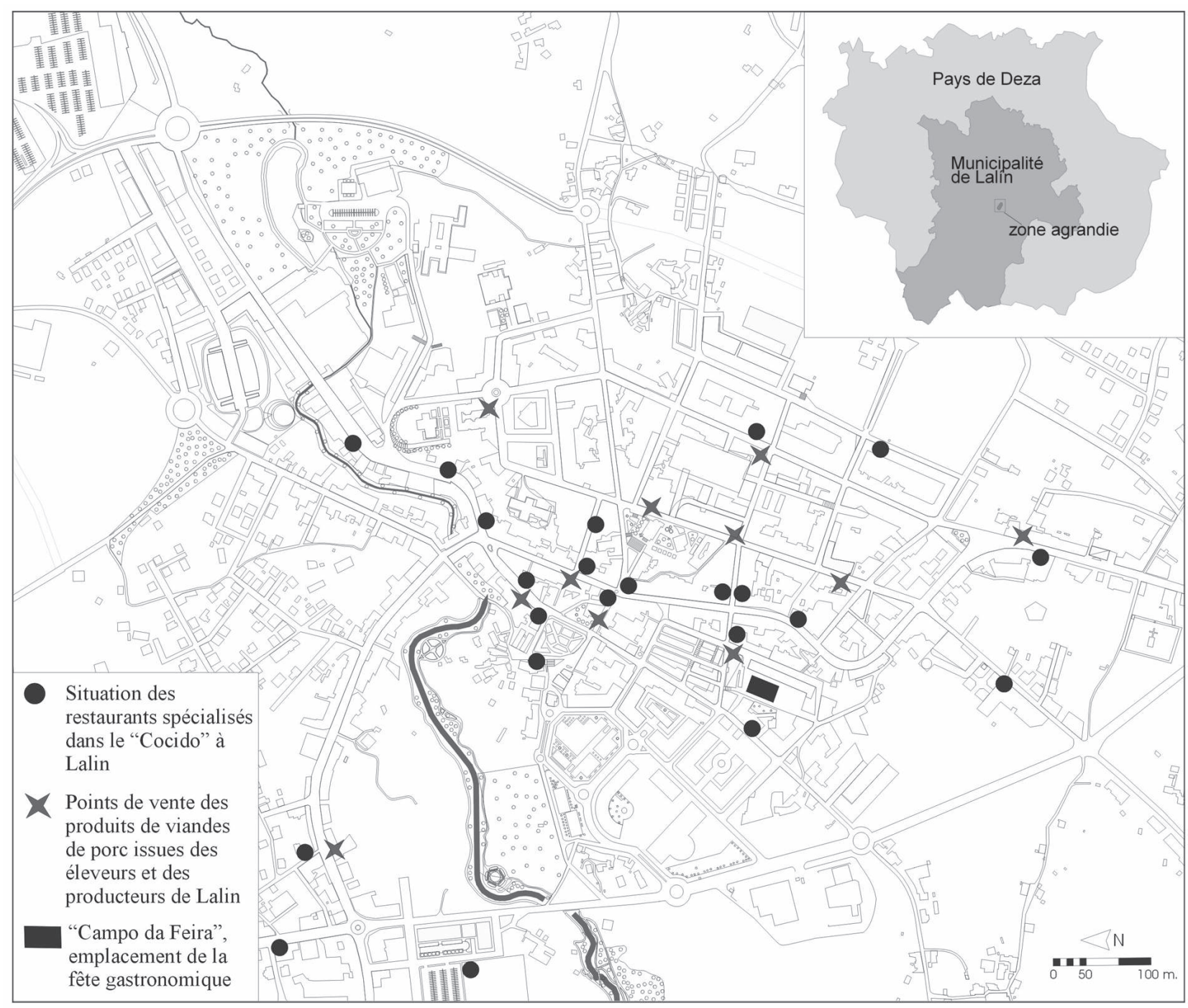

Figure 7 : Situation des restaurants spécialisés dans le cocido et les points de vente de viandes de porc issues des éleveurs et des producteurs de Lalín. D’autres établissements spécialisés se trouvent dans les zones rurales de la commune

Location of restaurants specializing in "cooked" and points of sale pig meat products from local producers in the town of Lalin. In rural areas of the municipality is located the other existing establishments

la place et le rôle du cocido dans leur activité et leur offre culinaire démontre que ce plat est un élément dynamisant de la restauration et par conséquent de l'économie locale (tableau 1).

Le pourcentage élevé des recettes liées à la vente de cocido établit une dépendance nette et une interrelation évidente entre producteur et restaurateurs. Le cocido possède un rôle non négligeable sur la dynamique des emplois durant le Mes del Cocido. Les restaurants enquêtés affirment qu'ils renforcent le nombre de travailleurs fixes à plus de 2 ou 3 professionnels pour faciliter le service au client. Si nous extrapolons ces données à la totalité de l'offre de restaurants de Lalín, nous pouvons estimer que le Mes del Cocido génère environ 50 à 60 postes de travail directs. L'origine locale des matières premières alimentaires utilisées a des répercussions sur la vie économique locale. Le cocido est capable d'attirer une clientèle touristique élargie à toute la Galice. La promotion du cocido est menée comme une vraie stratégie de marketing territorial qui, au cours de ces dernières années, a eu un franc succès auprès du public. Ceci se reflète dans le pourcentage de clients consommateurs de ce plat issus d'autres 


\begin{tabular}{|c|c|c|c|}
\hline & Restaurant I & Restaurant II & Restaurant III \\
\hline Nombre de plats de cocido servis par saison & 1600 & 3000 & 4200 \\
\hline Importance du cocido par rapport au chiffre d'affaire total du restaurant & $80 \%$ & $90 \%$ & $90 \%$ \\
\hline Nombre de personnel embauché pour le Mes del Cocido & 2 & 3 & 3 \\
\hline Origine locale des ingrédients utilisés dans l'élaboration du cocido & $95 \%$ & $100 \%$ & $100 \%$ \\
\hline Pourcentage de clients étrangers à la zone de Lalín & $80 \%$ & $90 \%$ & $75 \%$ \\
\hline Provenance des clients étrangers à la zone de Lalín & $\begin{array}{l}\text { De toute la } \\
\text { Galice }\end{array}$ & $\begin{array}{c}\text { De toute la } \\
\text { Galice }\end{array}$ & $\begin{array}{c}\text { De toute la } \\
\text { Galice }\end{array}$ \\
\hline Moyenne d'âge de la clientèle & Plus de 40 ans & $\begin{array}{c}\text { Entre } 30 \text { et } \\
60 \text { ans }\end{array}$ & Plus de 45 ans \\
\hline Niveau socio-économique de la clientèle & Moyen haut & Moyen & Moyen haut \\
\hline Pourcentage de réservations de groupes organisés & $60 \%$ & $70 \%$ & $60 \%$ \\
\hline
\end{tabular}

Tableau 1 : Résultats de l'enquête menée auprès de trois restaurants de Lalín. Enquête directe auprès de propriétaires de restaurants (réalisée en avril 2010)

The results of the survey carried out at three restaurants in Lalin

localités. Dans l'enquête, on constate que le nombre de visiteurs qui viennent à Lalín pour déguster le cocido est élevé. Il s'agit essentiellement d'un tourisme intérieur de la Galice, des zones urbaines et littorales qui est attiré par l'offre gastronomique et les diverses activités ludiques et festives associées au cocido. Nombreux sont ceux qui arrivent en groupes organisés pour passer une journée sur place. Les résultats de l'enquête montrent aussi qu'il s'agit d'une clientèle d'âge adulte et issue d'un niveau social moyen, parfois moyen haut (ce classement découle de l'appréciation directe des propriétaires des restaurants dont l'expérience professionnelle peut être fiable). Cette situation peut être attribuée au fait que ce mets fait appel à une forte tradition et que son prix moyen le réserve à un public d'adultes actifs.

L'activité de restauration liée à l'offre du cocido est donc bel et bien un facteur de développement local pour Lalín. Hormis la création d'emplois dans le réseau des restaurants, c'est un véritable support tant pour l'implantation d'entreprises agro-alimentaires de transformation des produits de la viande que pour le commerce et les services locaux grâce à l'afflux de touristes.

\section{Conclusion}

Dans le contexte où les ressources culinaires et vinicoles sont désormais évaluées pour leur valeur intrinsèque, leur ajustement aux nouvelles ten- dances de consommation culturelle et leur caractère symbolique leur permettent de devenir de véritables outils de développement local et régional(Grande, 2001). Du point de vue socioéconomique, elles permettent une distribution équitable des bénéfices à caractère économique, social et culturel dans les sociétés locales d'accueil. Ceci contribue à une amélioration de l'éducation, de la formation, et à la création de postes de travail et de revenus. Cette mise en valeur des ressources facilite de nouvelles opportunités pour de nombreux territoires, en particulier en zones rurales. La valorisation touristique de ces ressources culinaires et viticoles devient alors un élément important qui dynamise leur économie et leur culture (Csergo et Lemasson, 2009; Armesto et Gómez, 2006; Montero et al., 2001)

Le développement du tourisme gourmand permet à la fonction tertiaire spécialisée de revitaliser l'activité primaire en participant au maintien et à la valorisation de formes d'agriculture et d'élevage qui auraient pu disparaître. Il favorise la consolidation d'entreprises de transformation et la commercialisation de produits locaux tout en contribuant de façon décisive à la préservation des paysages. Il autorise la transmission et la conservation des recettes culinaires anciennes, par le renouvèlement de pratiques liées aux modes de production primaire (sacrifice du bétail, par exemple), par la création d'écoles où sont transmis les connaissances et les savoirs culinaires locaux et par l'établissement de formes de protection des aliments. 
Notre analyse a ainsi démontré le rôle joué par les restaurants de Lalín dans l'affirmation du rayonnement gastronomique du cocido ces dernières décennies. Nous pouvons en tirer les conclusions suivantes :

- l'existence d'une identification évidente entre cette petite ville de l'arrière-pays galicien et le mets. Ce lien étroit est né de la succession d'activités de valorisation et de promotion qui ont contribué à l'émergence de l'idée publique à l'échelle régionale que Lalín est la meilleure localité pour la dégustation du cocido;

- l'identification «Lalín-Cocido » est un produit socioculturel construit et lié aux différentes stratégies de valorisation et de promotion du mets qui ont permit, au-delà de la petite ville de Lalín, un ancrage régional du discours et de l'imaginaire;

- elle a entrainé l'émergence d'une image de qualité et positive qui a servi de tremplin à la création d'un réseau de restaurants locaux spécialisés dans l'offre de cette spécialité culinaire, stimulés essentiellement par une demande touristique issue de toute la Galice, et tout particulièrement des zones littorales;

- l'effet cocido a des répercussions sur le développement d'un réseau qualitatif de transformation et de commercialisation des viandes, de porc spécifiquement, encouragé par les restaurateurs et participant à leur dynamique d'ouverture et de développement;

- le réseau de restaurants locaux et d'entreprises de transformations de la viande entraînent le maintien et la création d'un grand nombre d'emplois et servent d'attraits aux visiteurs et aux touristes qui voient là un bon exemple de développement local basé sur l'identification d'une localité à un mets.

\section{Bibliographie}

Albet A., 2001. ¿Regiones singulares y regiones sin lugares? Reconsiderando el estudio de lo regional y lo local en el contexto de la geografía postmoderna, Boletín de la AGE (32), p. 35-53.

Aldrey Vázquez J.-A. et Lois González R.-C., 2010. Breve Xeografía de Galicia. A Nosa Terra, Vigo, 102 p.

Alonso-Logroño P., 2000. Localización de la industria textil en Galicia, dans La industria de la moda en Galicia, Lalín, IDEGA-Universidade de Santiago de Compostela, p. 55-71.

Armesto López X.-A. et Gómez Martín B., 2006. Tourism and Quality Agro-Food Products : an opportunity for the Spanish
Countryside dans Tijdschrift loor Economische en Sociale Geografie, 97 (2), p. 166-177.

Armesto López X.-A., Gómez Martín B., 2004. Productos agroalimentarios de calidad, turismo y desarrollo local : el caso del Priorat dans Cuadernos Geográficos, 34 (2004-1), p. 83-94.

Armesto López X.-A. et Pazos Otón M., 2010. Productos galegos de calidade e Gastronomía. ¿Un renacer para o Agro Galego? dans Pina H., Marques H., Devy-Vareta N. (dir.) 2010. Grandes Problemáticas do espaço Europeu. Norte de Portugal e Galiza. Porto, Universidade do Porto, p. 113-130.

BONIfACE P., 2003. Tasting tourism : travelling for food and drink, Aldershot, Ashgate, 176 p.

Calabuig J. et Ministral M., 1999. Manual de Geografía turística de España, Madrid, Editorial Síntesis, 494 p.

Cazes G., 1992. Fondements pour une géographie du tourisme et des loisirs, Paris, Bréal, 189 p.

Csergo J., Lemasson J.-P., 2009. Voyage en gastronomies. L'invention des capitales et des régions gourmandes, Paris, Autrement, $370 \mathrm{p}$.

Escudero-Gómez L.-E., 2003. Las fiestas gastronómicas, atractivo turístico y promoción de Galicia : La Feria do Cocido de Lalín, una visión a través de la prensa, dans Descubrindo, Revista de Investigación Deza, Lalín, Seminario de Estudios de Deza, vol 3, p. 351-365.

Etcheverria O., 2010. Quelques jalons pour la caractérisation d'une cuisine en lien au lieu : la cuisine in Geography and terroirs, Dans Contemporary French and Francophone Studies, Vol. 14, n², March 2010, p. 193-199,

Etcheverria O., 2009. San Sebastian : capitale gastronomique parmi les capitales gastronomiques? dans Csergo, J. et Lemason, J.-P., Voyages en gastronomies. L'invention des capitales et des régions goumandes, Paris, Autrement, coll. «Mondes en mutations », p. 171-182.

Fumey G., Etcheverria O., 2009. L'Atlas mondial des cuisines et des gastronomies, Paris, gener-bibliography IвarRa J., 2001. Análisis de la oferta de turismo cultural en España, Estudios Turísticos, $\mathrm{n}^{\circ}$ 150, p. 15-40.

Harvey D., 1990. The condition of postmodernity. An Enquiry into the origins of cultural change, Oxford, Basil Blackwell, $392 \mathrm{p}$.

Lemasson J.-P., 2006. Penser le tourisme gourmand, dans Teoros : revue de recherche en Tourisme, vol. 25, $\mathrm{n}^{\circ} 1$, p. 3-4.

López Silvestre F., Lois R.-C., 2007. From political construct to tourist souvenir - building the "National" landscape through advertising in Galicia (Spain), dans Social Geography Discussions, 3, p. 237-272.

MABY J., 2002. Paysage et imaginaire : l'exploitation de nouvelles valeurs ajoutées dans les terroirs viticoles, Annales de Géographie, 642, p. 198-211.

Mascarenhas R.-G., Gandara J.-M., 2010. Producción y transformación territorial. La gastronomía como atractivo turístico, dans Estudios y perspectivas en Turismo, Buenos Aires, Centro de Estudios y Perspectivas en Turismo, vol. 19, p. 776-791.

Montero I., Gutiérrez D., Díaz R., 2001. La cultura como componente de la oferta de los destinos turísticos maduros, dans Estudios Turísticos, n 150, p. 41-55. 
Moulin C., 2000. Gastronomy and tourism : Must every tourist wish be our command?, dans Revue $d u$ Tourisme, $\mathrm{n}^{\circ} 1$, p. 19-24.

Ritzer G., 1998. The McDonaldization Thesis : explorations and extensions, London, Ed. Sage, 212 p.

Rodriguez-Gonzalez R., 1994. Lalín. Un proceso de urbanización e desenvolvemento. 1960-1990, Deputación de Pontevedra, $177 \mathrm{p}$.

Rodríguez-González R., 2000. Pequeñas ciudades y sistemas productivos locales, dans La industria de la moda en Galicia,
Lalín, IDEGA-Universidade de Santiago de Compostela, p. 91-102.

Soja E., 1989. Postmodern geographies : the reassertion of space in critical social theory, London/New York, Verso, $222 \mathrm{p}$.

Terkenli T., 2002. Landscapes of tourism : towards a global cultural economy of space?, dans Tourism Geographies, 4(3), p. 227-254.

Unry, J., 2002. The Tourist Gaze, London/New Delhi, Thousand Oaks/SAGE. 